\title{
The clinical characteristics of COVID-19 patients with pneumonia in family clusters
}

\author{
Guangchaun Dai ${ }^{1}$, Yating $\mathrm{Gao}^{2}$, Likun Zhou ${ }^{3}$, Zhen Zhou ${ }^{3}$, Weiwei Gao ${ }^{1}$, Xiangrong \\ Zhang $^{1}$, Tingqiu $\mathrm{Ge}^{1}$, Yi Zeng ${ }^{1}$, Xia Zhang ${ }^{1}$, and Yongxiang $\mathrm{Yi}^{1}$ \\ ${ }^{1}$ The Second Hospital of Nanjing \\ ${ }^{2}$ Anhui University of Traditional Chinese Medicine \\ ${ }^{3}$ Nanjing University
}

November 16, 2020

\section{The clinical characteristics of COVID-19 patients with pneumonia in family clusters}

Guangchuan Dai ${ }^{1^{*}}$, Yating $\mathrm{Gao}^{2 *}$, Likun Zhou ${ }^{3,4^{*}}$, Zhen $\mathrm{Zhou}^{3^{*}}$, Weiwei Gao ${ }^{1}$, XiangrongZhang ${ }^{1}$, Tingqiu $\mathrm{Ge}^{1}$, Yi zeng ${ }^{1 \#}$, Xia Zhang ${ }^{1 \#}$, Yongxiang $\mathrm{Yi}^{1 \#}$

1Affiliated Hospital of Nanjing University of Chinese Medicine, The Second Hospital of Nanjing, Nanjing Municipal Public Health Medical Center, Nanjing, Jiangsu 210003, P.R. China

2Graduate School, Anhui University of Chinese Medicine, Hefei, Anhui 230012, P.R. China

3Nanjing Drum Tower Hospital Center of Molecular Diagnostic and Therapy, State Key Laboratory of Pharmaceutical Biotechnology, Jiangsu Engineering Research Center for MicroRNA Biology and Biotechnology, NJU Advanced Institute of Life Sciences (NAILS), NJU Institute of AI Biomedicine and Biotechnology, School of Life Sciences, Nanjing University, Nanjing 210023, P.R. China

4Tianjin Medical University Cancer Institute and Hospital, National Clinical Research Center for Cancer, Tianjin's Clinical Research Center for Cancer, Key Laboratory of Cancer Prevention and Therapy, Tianjin 300060, P.R. China

*These authors contributed equally

\# Correspondence: Yi Zeng(960559051@qq.com) or Xia Zhang(zhangxia365@sina.com) or Yongxiang Yi (ian0216@yeah.net)

Affiliated Hospital of Nanjing University of Chinese Medicine, The Second Hospital of Nanjing, Nanjing Municipal Public Health Medical Center, No.1, Kangfu Road, Jiangning District, Nanjing City, Jiangsu Province, P.R. China.

Tel:+86-025-85091652

Keywords: COVID-19 ; clinical characteristics ; family clusters

To the editor:

Approximately $2 \%$ of COVID-19 patients are younger than 20 years old ${ }^{1}$. Intrafamilial transmission is the main method of paediatric infection. The SARS-CoV-2 infection incidence in assessed paediatric patients increased from $1.6 \%$ to $12.3 \%$ with increased screening in Wuhan ${ }^{2}{ }^{3}$. Among them, $53 \%-65 \%$ of paediatric patients had pneumonia that required hospitalization ${ }^{4},{ }^{3}$. The time to become SARS-CoV-2 PCR negative (TTN) in paediatric patients without pneumonia was shorter than that of paediatric patients with 
pneumonia $^{4}$. Considering that the virulence of virus strains may vary between families while it is most likely constant among family members, analysis of paediatric patient characteristics, including TTN, in families will extend the understanding of familial virus infection. We analysed the clinical characteristics of paediatric patients (aged [?]18 years) and their familial adults (details are provided in the Supplementary Appendix).

After screening 93 patients, 4 paediatric and 9 adult patients in 4 families treated at the Nanjing Second Hospital from February 2020 to March 2020 were included (Fig. S1). Most patients were female. The median ages of the paediatric and adult groups were 10 and 47 years, respectively. Two families had a Wuhan exposure history. The most frequent antiviral drugs in paediatric patients were interferon $\alpha(4 / 4)$ and lopinavir/ritonavir (3/4). Three paediatric patients and 2 adults received concurrent antibiotics. Except for one paediatric patient who received azithromycin because of positive mycoplasma IgM, the other 4 patients (2 patients in each group) were treated with empirical antibiotics because of aggravated pneumonia. Most patients received glucocorticoids because of aggravated pneumonia (details in TABLE 1). The TTN in the paediatric group was longer than that in the adult group, using the paired $t$ test (median 16.5 days vs. 10.4 days, $\mathrm{P}<0.05$ ), while there was no difference between the two groups for the time to CT finding improvement $(\mathrm{P}>0.05)$ (Fig. S2).

This study showed that children with pneumonia need more time to recover than their familial adults even though children may be less susceptible to SARS-CoV-2. A large number of children should have been infected and have pneumonia in the global pandemic situation. As the time to CT finding improvement was not different between paediatric patients and their familial adults, children should not receive more frequent CT scans than adults. Considering our results and the fact that prolonged viral shedding occurs in the respiratory secretions and faeces of children ${ }^{5}$, additional attention should be paid to paediatric COVID-19 patients with pneumonia.

\section{Author contributions}

YY, XZ, YZ, GD and YG conceptualised the study design. GD, LZ and ZZ collected demographic, clinical, and laboratory data. WG and XRZ plotted the figure and tables. GD, YG, LZ and ZZ wrote the first draft. $\mathrm{XZ}, \mathrm{YZ}$ and YY commented on and revised the manuscript, WG, XZ, and TG analyzed the data. All authors read and approved the final report.

\section{Acknowledgements}

We are very appreciated to all the staff of the Nanjing second Hospital for their dedication in looking after the patients.

\section{Declaration of interests statement}

The authors have no competing interests to declare.

\section{References}

1. Wu Z, McGoogan JM. Characteristics of and Important Lessons From the Coronavirus Disease 2019 (COVID-19) Outbreak in China: Summary of a Report of 72314 Cases From the Chinese Center for Disease Control and Prevention. JAMA 2020.

2. Liu W, Zhang Q, Chen J, et al. Detection of Covid-19 in Children in Early January 2020 in Wuhan, China. N Engl J Med 2020;382(14):1370-1371.

3. Lu X, Zhang L, Du H, et al. SARS-CoV-2 Infection in Children. N Engl J Med 2020;382(17):1663-1665.

4. Qiu H, Wu J, Hong L, Luo Y, Song Q, Chen D. Clinical and epidemiological features of 36 children with coronavirus disease 2019 (COVID-19) in Zhejiang, China: an observational cohort study. Lancet Infect Dis 2020;20(6):689-696.

5. Cai J, Xu J, Lin D, et al. A Case Series of children with 2019 novel coronavirus infection: clinical and epidemiological features. Clin Infect Dis. 2020. 


\section{Hosted file}

2020-11-14 Table.pdf available at https://authorea.com/users/375991/articles/493156-theclinical-characteristics-of-covid-19-patients-with-pneumonia-in-family-clusters 